\title{
Effect of a hospital policy of not accepting free infant formula on in-hospital formula supplementation rates and breast-feeding duration
}

\author{
Marie Tarrant ${ }^{1, *}$, Kris YW Lok ${ }^{1}$, Daniel YT Fong ${ }^{1}$, Irene LY Lee ${ }^{2}$, Alice Sham ${ }^{3}$, \\ Christine Lam ${ }^{4}$, Kendra M Wu ${ }^{5}$, Dorothy L Bai ${ }^{1}$, Ka Lun Wong ${ }^{1}$, Emmy MY Wong ${ }^{6}$, \\ Noel PT Chan ${ }^{1}$ and Joan E Dodgson ${ }^{7}$ \\ ${ }^{1}$ School of Nursing, Li Ka Shing Faculty of Medicine, The University of Hong Kong, 4/F William M.W. Mong Block, \\ 21 Sassoon Road, Hong Kong Special Administrative Region (SAR), People's Republic of China: ${ }^{2}$ Queen Mary \\ Hospital, Hong Kong SAR, People's Republic of China: ${ }^{3}$ United Christian Hospital, Kowloon, Hong Kong SAR, \\ People's Republic of China: ${ }^{4}$ Department of Obstetrics and Gynaecology, Queen Elizabeth Hospital, Kowloon, Hong \\ Kong SAR, People's Republic of China: ${ }^{5}$ School of Public Health, Li Ka Shing Faculty of Medicine, The University of \\ Hong Kong, Hong Kong SAR, People's Republic of China: 'Department of Health and Physical Education, The Hong \\ Kong Institute of Education, New Territories, Hong Kong SAR, People's Republic of China: ${ }^{7}$ College of Nursing and \\ Healthcare Innovation, Arizona State University, Phoenix, AZ, USA
}

Submitted 26 September 2014: Final revision received 15 December 2014: Accepted 7 January 2015: First published online 27 February 2015

\begin{abstract}
Objective: To investigate the effect of public hospitals in Hong Kong not accepting free infant formula from manufacturers on in-hospital formula supplementation rates and breast-feeding duration.

Design: Prospective cohort study.

Setting: In-patient postnatal units of four public hospitals in Hong Kong.

Subjects: Two cohorts of breast-feeding mother-infant pairs ( $n$ 2560). Cohort 1 ( $n$ 1320) was recruited before implementation of the policy to stop accepting free infant formula and cohort 2 ( $n$ 1240) was recruited after policy implementation. Participants were followed prospectively for 12 months or until they stopped breast-feeding.

Results: The mean number of formula supplements given to infants in the first $24 \mathrm{~h}$ was $2.70(\mathrm{sD} 3 \cdot 11)$ in cohort 1 and 1.17 (SD 1.94) in cohort $2(P<0.001)$. The proportion of infants who were exclusively breast-fed during the hospital stay increased from $17.7 \%$ in cohort 1 to $41.3 \%$ in cohort $2(P<0.001)$ and the risk of breast-feeding cessation was significantly lower in cohort 2 (hazard ratio $=0.81$; $95 \%$ CI $0.73,0 \cdot 90)$. Participants who non-exclusively breast-fed during the hospital stay had a significantly higher risk of stopping any or exclusive breast-feeding. Higher levels of formula supplementation also increased the risk of breast-feeding cessation in a dose-response pattern.

Conclusions: After implementation of a hospital policy to pay market price for infant formula, rates of in-hospital formula supplementation were reduced and the rates of in-hospital exclusive breast-feeding and breast-feeding duration increased.
\end{abstract}

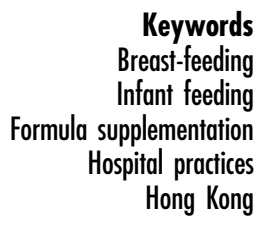

Keywords

Breast-feeding

Infant feeding

spital practices

Hong Kong
The recommendation for exclusive breast-feeding for the first 6 months of life is based on the nutritional and immunological importance of breast-feeding for optimal health, growth and development ${ }^{(1,2)}$ and the welldocumented risks of infant formula feeding ${ }^{(3,4)}$. Although breast-feeding initiation rates have increased substantially in most developed countries over the past several decades, early breast-feeding cessation is common and rates of exclusive breast-feeding remain low ${ }^{(5,6)}$. Many healthy breast-feeding newborns are supplemented with infant formula before leaving hospital, with rates ranging from $23 \%$ to $82 \%{ }^{(7-13)}$. Supplements are often given for non-medical reasons ${ }^{(14)}$ such as maternal fatigue, instrumental or operative deliveries, and perceived insufficient milk $^{(7,8,11)}$. Early infant formula supplementation disrupts the establishment of breast-feeding by reducing the 
frequency of breast-feeding and the amount of breast milk removed from the breasts, which often necessitates further supplementation $^{(15)}$.

Excessive marketing and promotion of infant formula, including the provision of free or heavily discounted products to hospitals and medical providers, may contribute to the high rates of in-hospital formula supplementation and the subsequent reduced rates of exclusive breastfeeding ${ }^{(16)}$. In 1989, the WHO and UNICEF developed a set of guidelines for maternity care facilities to protect, promote and support breast-feeding ${ }^{(17)}$. The WHO later launched the Baby Friendly Hospital Initiative (BFHI), which outlined minimum global criteria for accreditation as a Baby-Friendly Hospital. The BFHI stipulates that hospitals should not accept free or heavily discounted infant formula products from manufacturers and must pay market price for all breast-milk substitutes ${ }^{(18)}$. Despite this BFHI guideline, financial incentives mean many hospitals that are not BFHI accredited continue to accept infant formula products from the manufacturers ${ }^{(16)}$. Research has highlighted that early infant formula supplementation is one of the strongest predictors of early breast-feeding cessation ${ }^{(8,11,19-21)}$. Providing mothers with infant formula samples upon hospital discharge has also been shown to shorten breast-feeding duration $^{(22,23)}$. It is widely believed that hospitals' acceptance of free infant formula encourages non-medically indicated supplementation ${ }^{(24,25)}$. Although a number of studies have found that the BFHI improves hospital practices $^{(26-28)}$ and breast-feeding outcomes ${ }^{(21,29,30)}$, none have specifically examined the impact of hospitals stopping the acceptance of free infant formula. This suggests that there is no direct evidence to support this belief. Given the pervasiveness of infant formula promotion globally, it would be beneficial to have further evidence of the benefits to breast-feeding mothers and infants when hospitals stop the acceptance of free infant formula.

Breast-feeding initiation rates in Hong Kong are above $85 \%{ }^{(31)}$. However, overall breast-feeding duration remains short and rates of exclusive breast-feeding are low ${ }^{(32)}$. Historically, all Hong Kong hospitals have accepted free infant formula from manufacturers. In 2006, all public and most private hospitals adopted a policy to purchase infant formula at market price as a step towards becoming more baby-friendly. This was a significant change and provided a unique opportunity to study the effect of the policy on hospital practices and breast-feeding outcomes. The present study aimed to assess the effect of stopping free infant formula in public hospitals on: (i) the number of supplemental feeds given to breast-feeding babies during their hospital stay; (ii) the proportion of infants who were given formula supplements during their hospital stay; and (iii) the overall duration and exclusivity of breastfeeding. Additionally, in a pooled analysis we aimed to assess the overall effect of the amount of in-hospital infant formula supplementation on the duration of any or exclusive breast-feeding.

\section{Methods}

\section{Design, setting and participants}

The present study employed a prospective cohort design. Two cohorts of breast-feeding mothers in the immediate postpartum period were recruited from four public hospitals in Hong Kong, one before and the second after the change in the infant formula policy. All Hong Kong residents have access to free, high-quality antenatal, postnatal and well-child health care, and maternal and infant mortality rates are some of the lowest in the world ${ }^{(33)}$. Hong Kong has eight public and ten private hospitals that offer obstetric services, none of which are BFHI accredited. The normal length of postnatal stay in public hospitals is approximately $48 \mathrm{~h}$ for a vaginal birth and $72 \mathrm{~h}$ for a caesarean birth. The Hong Kong Hospital Authority, a subvented government organization, manages all public hospitals; while private hospitals are managed by various charitable, philanthropic and religious organizations. Public hospitals accounted for $67 \cdot 8 \%$ of all births to Hong Kong mothers in $2011^{(34)}$. Over the past decade, a high proportion of patients who gave birth in private hospitals in Hong Kong were birth tourists from Mainland China ${ }^{(35)}$. Participant recruitment was therefore limited to public hospitals.

The Hospital Authority proposed the infant formula policy change in 2006, with implementation planned for 2007. However, other constraints on the obstetrics services in Hong Kong hospitals ${ }^{(35)}$ meant implementation was delayed until April 2010. The first cohort of breast-feeding mothers (cohort 1) was recruited in 2006-2007, before the planned implementation of the new infant formula policy, and served as the pre-policy baseline group. Following the policy implementation, a second cohort of breast-feeding mothers (cohort 2) was recruited in 2011-2012, as the post-policy group. All study protocols, including selection criteria, participant recruitment, data collection procedures and follow-up intervals, were exactly the same for both cohorts. Eligibility criteria were: (i) an intention to breastfeed; (ii) a singleton pregnancy; (iii) Cantonese speaking; and (iv) no serious medical or obstetric complications. Further exclusion criteria for the newborn were: (i) less than 37 weeks' gestation; (ii) a 5 min Apgar score less than 8; (iii) birth weight less than $2500 \mathrm{~g}$; (iv) severe medical conditions or congenital malformations; (v) admission to the special care baby unit for $48 \mathrm{~h}$ or more after delivery; or (vi) admission to the neonatal intensive care unit after delivery.

\section{Data collection}

Data were collected directly from participants while in hospital and through telephone follow-up after discharge. Data collection included: (i) baseline demographic data; (ii) maternal and infant data; (iii) in-hospital infant feeding data; (iv) follow-up infant feeding data at 1, 2, 3, 6, 9 and 12 months postpartum or until the participant 
stopped breast-feeding completely; and (v) final breastfeeding status data.

\section{Study variables}

During the postnatal hospitalization, infants were considered exclusively breast-fed if they received no solids, no breast-milk substitutes and no water or other liquids (other than vitamins or medications) ${ }^{(36)}$. For nonexclusively breast-feeding infants, the proportion of total daily feeds that were breast milk was computed. As most new mothers were discharged after $48 \mathrm{~h}$, in-hospital feeding data were collected for the first $48 \mathrm{~h}$ of admission. Non-exclusively breast-fed infants supplemented with infant formula or other milk substitutes were classified as high-partial breast-feeding $(>80-<100 \%$ of feeds were breast-feeds), medium-partial breast-feeding (20-80\% of feeds were breast-feeds) or low-partial breastfeeding $\left(<20 \%\right.$ of feeds were breast-feeds) ${ }^{(37)}$.

After hospital discharge, breast-feeding status was assessed at the intervals outlined above through telephone interviews. Participants were asked about the amount and type of feedings given to the infant in the $24 \mathrm{~h}$ period before the interview ${ }^{(36)}$. Infants were categorized as exclusive breast-feeding, partial breast-feeding or exclusive formula feeding. Exclusive breast-feeding was defined according to the definition outlined above ${ }^{(36)}$. If the participant had stopped breast-feeding altogether during that follow-up interval, she was asked to report the total duration of any and exclusive breast-feeding in weeks. As the present study aimed to examine the effect of in-hospital formula supplementation on the duration of any and exclusive breast-feeding, exclusive breastfeeding duration was computed based on how the mother reported feeding her baby after leaving the hospital and did not include in-hospital formula supplementation $^{(38)}$.

\section{Sample size calculation}

The sample size calculation was based on primary comparisons of the duration of exclusive or any breast-feeding over a 12-month period. To account for multiplicity, the nominal level of significance for calculating the sample size for each outcome was taken as $2.5 \%$ (by Bonferroni adjustment) to control the overall false positive error rate to within $5 \%$. The results of a previous Hong Kong study ${ }^{(39)}$ indicated the median duration of exclusive breast-feeding was 3.5 weeks and that of any breastfeeding was 6 weeks. Based on other research ${ }^{(40)}$, an increase of 1.5 weeks in the median duration of both exclusive and any breast-feeding was estimated to indicate a clinically significant improvement in breast-feeding outcomes. This means that to detect such a difference with $80 \%$ power by a log-rank test, 170 women per group were needed to compare exclusive breast-feeding and 510 women per group to compare any breast-feeding. Factoring in a loss to follow-up rate of $25 \%$, approximately
700 to 800 mother-infant pairs were needed in each cohort to obtain a final sample size of 510 per group.

\section{Statistical analyses}

Descriptive statistics were used to compare the sociodemographic characteristics of the two study cohorts. The effect of the policy on in-hospital formula supplementation rates and on overall patterns of breast-feeding was assessed using $t$ tests for continuous variables and $\chi^{2}$ tests for categorical variables. Kaplan-Meier survival curves were constructed to compare the overall duration of any or exclusive breast-feeding between the two cohorts and to assess any dose-response relationship between the amount of in-hospital formula supplementation and the time to cessation of any or exclusive breast-feeding in the total sample. All Kaplan-Meier survival curves were compared using a log-rank test of equality or a trend test of the survival functions, whichever was appropriate ${ }^{(41)}$. Finally, to assess independent associations between the infant formula policy, the amount of in-hospital formula supplementation and the duration of any or exclusive breast-feeding, unadjusted and fully adjusted multivariable Cox proportional hazards regression analyses were performed $^{(42)}$. The proportional hazards assumption was tested by assessing the log-log plots of estimated survival curves. For the multivariable analyses, three models were constructed with potential confounders added in turn based on subject matter relevance ${ }^{(43)}$. Model 1 adjusted for key sociodemographic variables (maternal age and education, family income, length of residence in Hong Kong, returning to work postpartum, study site); Model 2 further adjusted for maternal and birth data (parity, attendance at antenatal childbirth and breast-feeding classes, delivery type); and Model 3 further adjusted for breast-feeding-related variables (intention to exclusively breast-feed, participant's own breast-feeding status, previous breast-feeding experience, husband's infant feeding preference). The variables entered into the multivariable models were selected because they were factors that have been shown to significantly affect breast-feeding duration in this population ${ }^{(32,39)}$ or they were characteristics that differed significantly between the two study cohorts $(P<0.05)$. In multivariable models using the pooled sample of participants, further adjustment was made for study cohort. For all analyses, participants who were lost to follow-up were censored at the time of last contact. All data were analysed using the Stata statistical software package version $13 \cdot 1$, with a $5 \%$ level of significance used in all statistical tests.

\section{Results}

From June 2006 to July 2007, 1417 mother-infant pairs were recruited from four study sites to form the cohort 1 baseline sample. Following policy implementation in April 
2010, a second cohort of 1287 mother-infant pairs was recruited from the same four study sites. A total of 2560 mother-infant pairs were included in the final analysis (Fig. 1).

The sociodemographic characteristics of both cohorts are presented in Table 1 . There were no differences in participant age and education levels of the two cohorts. Participants in cohort 2 were more likely to be multiparous $(P=0.012)$, have previous breast-feeding experience $(P=0.001)$ and be planning to exclusively breast-feed $(P<0.001)$. Cohort 2 participants were also less likely to have attended antenatal childbirth and breast-feeding classes and be intending to return to work within 6 months postpartum (all $P<0.001$ ). The husbands of participants in cohort 2 were less likely to explicitly prefer exclusive breast-feeding for the infant and more likely to have no particular infant feeding preference (both $P<0 \cdot 001$ ).

The mean number of infant formula supplements given to infants in the first $24 \mathrm{~h}$ of life was 2.70 (SD 3.11) before policy implementation and 1.17 (SD 1.94) after implementation $(P<0 \cdot 001)$. In cohort 1 , the mean proportion of breast-milk feeds given to infants in the first $24 \mathrm{~h}$ of life, in the second $24 \mathrm{~h}$ of life and for the total hospital stay was 64.4 (SD 34.7) \%, 72.0 (SD 27.0) \% and 68.2 (SD 27.0) \%, respectively. In cohort 2 , the corresponding mean proportions of breast-milk feeds given to infants were 83.3 (SD 29.1) \%, 86.2 (sD 23.0) \% and 84.2 (sD 23.2) \%, respectively. All comparisons were statistically significant at a level of $P<0.001$. After implementation of the infant formula policy, more mothers ( $41.3 \% v \cdot 17.7 \%)$ exclusively breastfed during hospitalization (Fig. 2).

The median duration of breast-feeding increased from 8 to 12.5 weeks after policy implementation. Figure 3 shows that the risk of breast-feeding cessation was significantly lower after the policy implementation $(P<0 \cdot 001)$, but that there was no significant change in the risk of stopping exclusive breast-feeding $(P=0 \cdot 58)$. Even after adjusting for potential confounding variables (Table 2 ), infants in cohort 2 had an approximately $20 \%$ lower risk of cessation of any breast-feeding (hazard ratio $(\mathrm{HR})=0.81 ; 95 \% \mathrm{CI} 0 \cdot 73,0 \cdot 90$ ) but there was no change in the risk of stopping exclusive breast-feeding ( $\mathrm{HR}=1 \cdot 01 ; 95 \% \mathrm{CI} 0.93,1 \cdot 11)$.

In the pooled sample, infants who were supplemented with infant formula had an increased risk of cessation of any and exclusive breast-feeding with higher levels of supplementation than infants who received no in-hospital formula supplementation (exclusive breast-feeding; Fig. 4). In the fully adjusted model, infants who received highpartial breast-feeding during the hospital stay $(<20 \%$ infant formula) had an approximately $30 \%$ increased risk of breast-feeding cessation ( $\mathrm{HR}=1 \cdot 29$; $95 \%$ CI 1.15, 1.46) and infants who received medium-partial (20-80\% infant formula) and low-partial breast-feeding (>80\% infant formula) had a $68 \%$ to $73 \%$ increased risk discontinuing breast-feeding (Table 3) than infants who were exclusively breast-fed. The effect of in-hospital formula supplementation on the overall duration of exclusive breastfeeding was similar. Infants who were high-, medium- and low-partially breast-fed had an increased risk of exclusive breast-feeding cessation of approximately $20 \%, 50 \%$ and $70 \%$, respectively. The log-log plots for all Cox regression models showed that the proportional-hazards assumption was not violated.

\section{Discussion}

After public hospitals in Hong Kong implemented a policy of paying market price for infant formula, in-hospital formula supplementation of breast-feeding babies decreased

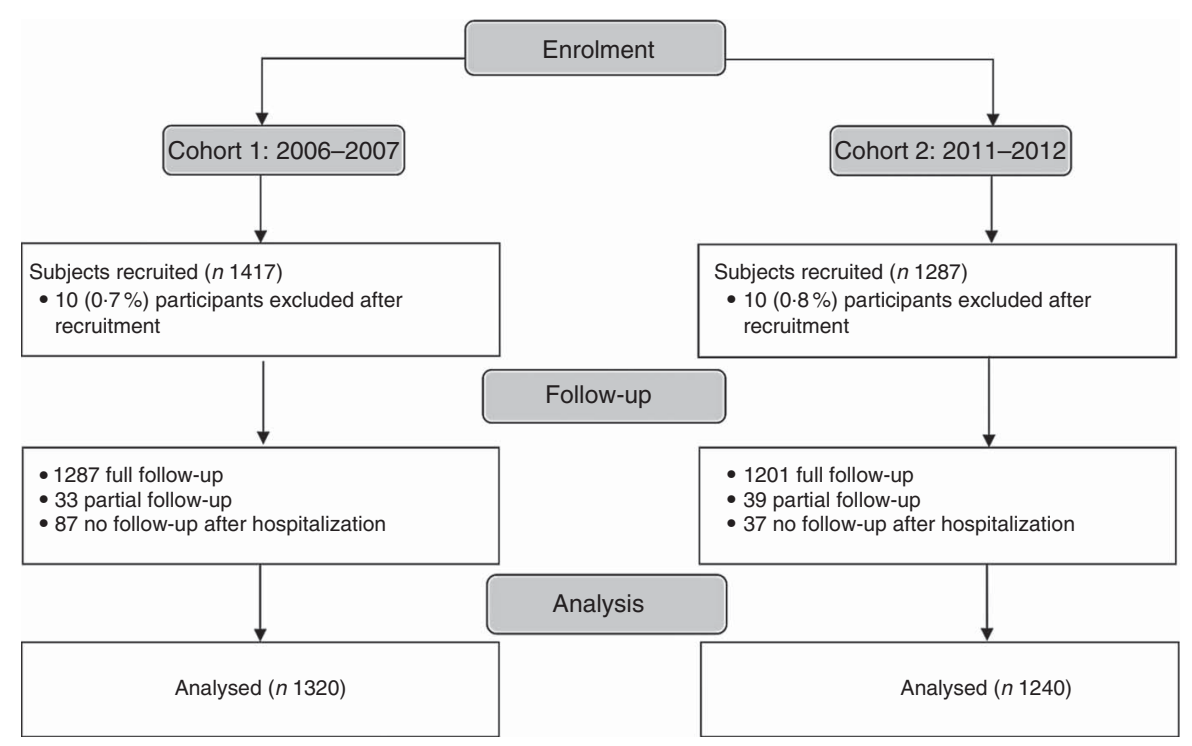

Fig. 1 Participant recruitment flow diagram 
Table 1 Comparison of the characteristics of the two study cohorts of breast-feeding mother-infant pairs, who were recruited from in-patient postnatal units of four public hospitals in Hong Kong, before (cohort 1) and after (cohort 2) implementation of the policy to stop accepting free infant formula

\begin{tabular}{|c|c|c|c|c|c|c|c|}
\hline \multirow[b]{2}{*}{ Demographic variable } & \multicolumn{2}{|c|}{ Total $(n 2560)$} & \multicolumn{2}{|c|}{ Cohort 1 (n 1320) } & \multicolumn{2}{|c|}{ Cohort 2 ( $n$ 1240) } & \multirow[b]{2}{*}{$P$ value } \\
\hline & $n$ & $\%$ & $n$ & $\%$ & $n$ & $\%$ & \\
\hline Maternal age & & & & & & & 0.193 \\
\hline $18-24$ years & 161 & $6 \cdot 3$ & 88 & $6 \cdot 7$ & 73 & 5.9 & \\
\hline $25-29$ years & 611 & 23.9 & 303 & 23.0 & 308 & 24.5 & \\
\hline $30-34$ years & 1138 & 44.5 & 609 & $46 \cdot 1$ & 529 & $42 \cdot 7$ & \\
\hline$\geq 35$ years & 650 & $25 \cdot 4$ & 320 & $24 \cdot 2$ & 330 & $26 \cdot 6$ & \\
\hline Maternal education & & & & & & & 0.181 \\
\hline Primary & 78 & $3 \cdot 1$ & 47 & 3.6 & 31 & 2.5 & \\
\hline Secondary & 1464 & $57 \cdot 2$ & 763 & 57.8 & 701 & 56.5 & \\
\hline University degree or above & 1018 & 39.8 & 510 & 38.6 & 508 & $41 \cdot 0$ & \\
\hline Monthly family income (HKD)* & & & & & & & 0.004 \\
\hline$<\$ 15000$ & 499 & $19 \cdot 8$ & 287 & 22.4 & 212 & $17 \cdot 1$ & \\
\hline$\$ 15000-29999$ & 873 & $34 \cdot 6$ & 430 & 33.5 & 443 & $35 \cdot 7$ & \\
\hline$\geq \$ 30000$ & 1152 & $45 \cdot 6$ & 567 & $44 \cdot 2$ & 585 & $47 \cdot 2$ & \\
\hline Length of residence in Hong Kong & & & & & & & $<0.001$ \\
\hline$<5$ years & 239 & $9 \cdot 3$ & 147 & $11 \cdot 1$ & 92 & 7.4 & \\
\hline 5 to $\geq 15$ years & 740 & 28.9 & 329 & 24.9 & 411 & 33.2 & \\
\hline Since birth & 1581 & 61.8 & 844 & 63.9 & 737 & 59.4 & \\
\hline Return to work $\leq 6$ months postpartum & & & & & & & $<0.001$ \\
\hline No & 788 & $30 \cdot 8$ & 347 & $26 \cdot 3$ & 441 & $35 \cdot 6$ & \\
\hline Yes & 1772 & $69 \cdot 2$ & 973 & 73.7 & 799 & $64 \cdot 4$ & \\
\hline Parity & & & & & & & 0.012 \\
\hline Primiparous & 1471 & 57.5 & 790 & $59 \cdot 9$ & 681 & $54 \cdot 9$ & \\
\hline Multiparous & 1089 & 42.5 & 530 & $40 \cdot 2$ & 559 & $45 \cdot 1$ & \\
\hline Attended antenatal childbirth class & & & & & & & $<0.001$ \\
\hline No & 1140 & 44.5 & 459 & $34 \cdot 8$ & 681 & 54.9 & \\
\hline Yes & 1420 & 55.5 & 861 & $65 \cdot 2$ & 559 & $45 \cdot 1$ & \\
\hline Attended antenatal breast-feeding class & & & & & & & $<0.001$ \\
\hline No & 1440 & $56 \cdot 3$ & 614 & $46 \cdot 5$ & 826 & $66 \cdot 6$ & \\
\hline Yes & 1120 & 43.8 & 706 & 53.5 & 414 & 33.4 & \\
\hline Delivery type & & & & & & & $<0.001$ \\
\hline Spontaneous vaginal delivery $\dagger$ & 1929 & $75 \cdot 4$ & 953 & $72 \cdot 2$ & 976 & $78 \cdot 7$ & \\
\hline Assisted vaginal delivery & 164 & $6 \cdot 4$ & 104 & 7.9 & 60 & $4 \cdot 8$ & \\
\hline Planned caesarean delivery & 217 & 8.5 & 114 & 8.6 & 103 & 8.3 & \\
\hline Emergency caesarean delivery & 250 & $9 \cdot 8$ & 149 & $11 \cdot 3$ & 101 & 8.2 & \\
\hline Planning to exclusively breast-feed & & & & & & & $<0.001$ \\
\hline No & 745 & $29 \cdot 1$ & 474 & 35.9 & 271 & 21.9 & \\
\hline Yes & 1815 & $70 \cdot 9$ & 846 & $64 \cdot 1$ & 969 & 78.2 & \\
\hline Participant breast-fed as a child & & & & & & & 0.039 \\
\hline No & 1435 & $56 \cdot 1$ & 714 & $54 \cdot 1$ & 721 & $58 \cdot 2$ & \\
\hline Yes & 1125 & $44 \cdot 0$ & 606 & 45.9 & 519 & 41.9 & \\
\hline Previous breast-feeding experience & & & & & & & 0.001 \\
\hline No & 1621 & 63.3 & 877 & $66 \cdot 4$ & 744 & $60 \cdot 0$ & \\
\hline Yes & 939 & $36 \cdot 7$ & 443 & 33.6 & 496 & $40 \cdot 0$ & \\
\hline Husband's infant feeding preference & & & & & & & $<0.001$ \\
\hline Breast-feeding only & 1343 & $52 \cdot 5$ & 815 & 61.7 & 528 & $42 \cdot 6$ & \\
\hline Infant formula and mixed feeding & 340 & $13 \cdot 3$ & 255 & $19 \cdot 3$ & 85 & 6.9 & \\
\hline No preference & 877 & $34 \cdot 3$ & 250 & 18.9 & 627 & 50.6 & \\
\hline Study site & & & & & & & $<0.001$ \\
\hline Hospital A & 716 & 28.0 & 394 & $29 \cdot 9$ & 322 & $26 \cdot 0$ & \\
\hline Hospital B & 609 & 23.8 & 339 & $25 \cdot 7$ & 270 & $21 \cdot 8$ & \\
\hline Hospital C & 627 & 24.5 & 290 & $22 \cdot 0$ & 337 & $27 \cdot 2$ & \\
\hline Hospital D & 608 & 23.8 & 297 & 22.5 & 311 & $25 \cdot 1$ & \\
\hline
\end{tabular}

HKD, Hong Kong Dollars.

*\$US $1=$ \$HK 7.78 .

†Spontaneous vaginal delivery refers to a birth without the use of drugs or other methods to induce labour and delivery, and a birth without the use of forceps or vacuum extraction.

significantly, the overall risk of breast-feeding cessation decreased and the median duration of breast-feeding increased from 8 to 12.5 weeks. Overall, breast-feeding duration was significantly longer after policy implementation, even after adjusting for confounding variables.
However, the overall duration of exclusive breast-feeding was unchanged, despite the finding that significantly more participants in cohort 2 had intended to exclusively breast-feed. Over the first 6 months, only about onehalf of all breast-feeding mothers in both cohorts were 


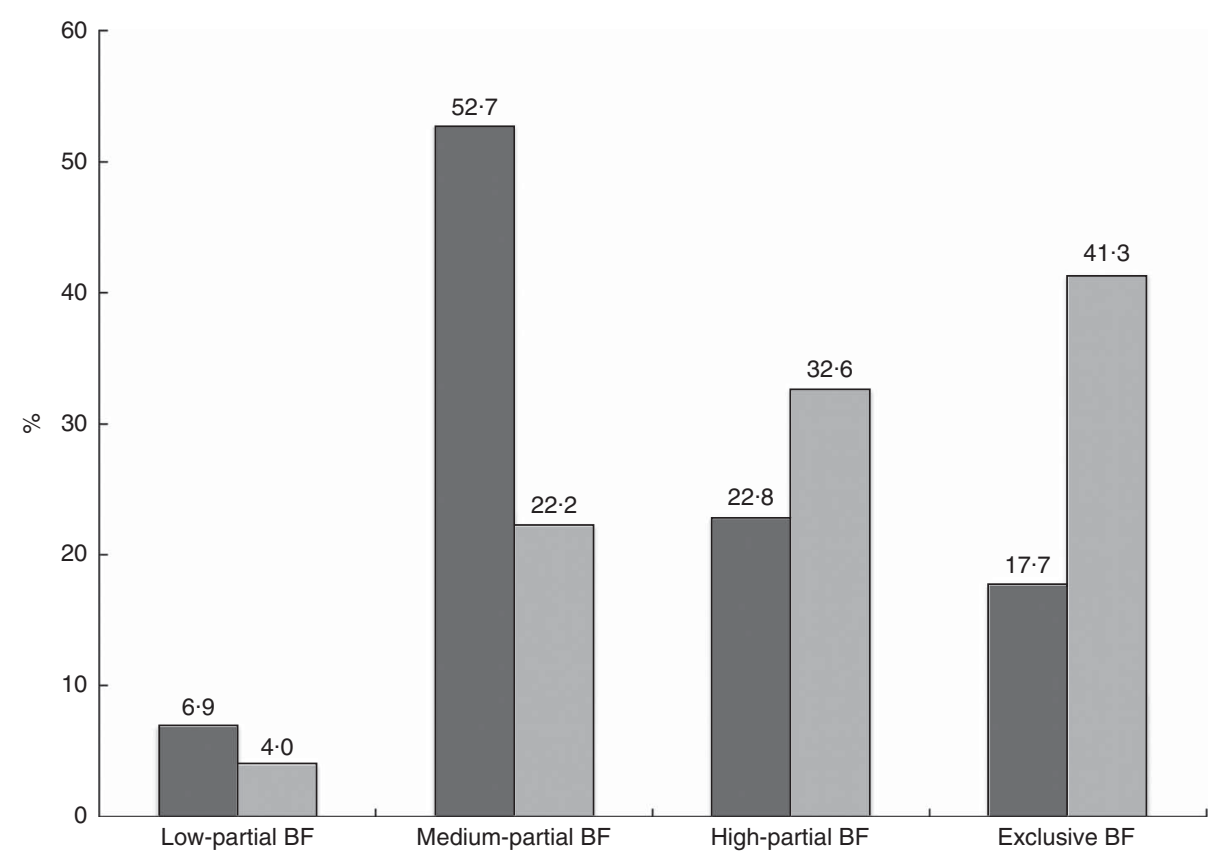

Fig. 2 In-hospital pattern of breast-feeding (BF) by study cohort ( $\square$, cohort 1, $n$ 1320; $\square$, cohort 2, $n$ 1240). The two cohorts of breast-feeding mother-infant pairs were recruited from in-patient postnatal units of four public hospitals in Hong Kong, before (cohort 1) and after (cohort 2) implementation of the policy to stop accepting free infant formula; all $P<0.001$

breast-feeding exclusively. Similarly, Feldman-Winter et $a l^{(23)}$ also found that removing free infant formula samples from discharge packs given to new mothers increased overall breast-feeding duration but failed to increase exclusive breast-feeding duration. Although breast-feeding intention is a strong predictor of the overall duration of any breast-feeding ${ }^{(44,45)}$, exposure to hospital practices that are consistent with the BFHI has been shown to strongly affect women's achievement of their exclusive breast-feeding goals ${ }^{(38,46)}$. Thus, further improvement in hospital practices that support breast-feeding would likely help to improve exclusive breast-feeding rates. It is also possible that breast-feeding mothers do not receive enough support once back in their communities ${ }^{(47)}$ and formula supplements provide reassurance to anxious new parents ${ }^{(48)}$. High rates of maternal employment may also affect rates of exclusive breast-feeding, with infant formula often introduced in preparation for a return to work ${ }^{(49)}$. In addition, infant formula companies have successfully perpetuated and reinforced the now widely accepted myth that many mothers do not produce sufficient breast milk to exclusively breast-feed and that some formula supplementation is required to meet infant nutritional needs ${ }^{(16)}$.

The present study highlighted that infants supplemented during postpartum hospitalization are substantially less likely to breast-feed at any time point compared with exclusively breast-fed infants. Evidence of a doseresponse relationship between the amount of in-hospital formula supplementation and the overall duration of any or exclusive breast-feeding was also found; a finding that has not previously been shown. This pattern suggests that protecting infants from formula supplementation in the early postpartum period may substantially improve breast-feeding duration, which is consistent with previous research ${ }^{(50-52)}$. However, some researchers have suggested that early supplementation with infant formula may be an indicator rather than a cause of breast-feeding difficulties ${ }^{(53)}$. Nevertheless, infant formula supplementation occurs too frequently in healthy breast-feeding newborns and even after implementation of the policy, almost $60 \%$ of infants received formula before hospital discharge. Few infants in the present study would meet the criteria for medically indicated supplementation ${ }^{(14,54)}$ as they were all full-term, healthy newborns without any major medical complications. Many supplements were given in the first $24 \mathrm{~h}$ after birth, earlier than most medical indications for supplementation would be apparent (i.e. excess weight loss, delayed lactogenesis, hyperbilirubinaemia or significant dehydration) ${ }^{(14)}$. Therefore, further reductions in the in-hospital formula supplementation rates of breastfeeding newborns are required to meet the BFHI standard of only providing supplements when medically necessary. In addition, new mothers and health workers should be clearly informed of the health risks associated with formula supplements and the negative effect of these supplements on breast-feeding continuation ${ }^{(55)}$.

The infant formula policy implementation was widely discussed and publicized during the extended interval between the decision and the actual implementation. Although there were no other major breast-feeding policy changes implemented in public hospitals during the 
interval between data collection, there were many discussions at the institutional level about how to make public hospitals more baby-friendly and about the process

(a)

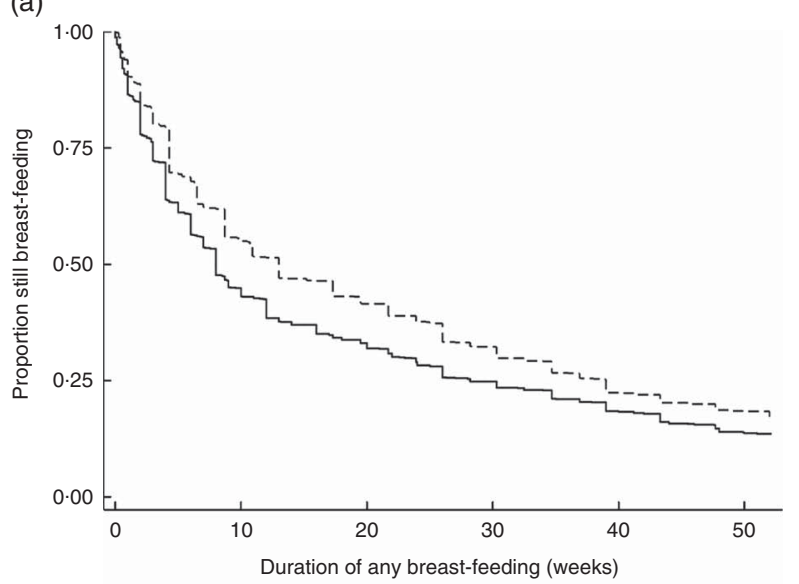

(b)

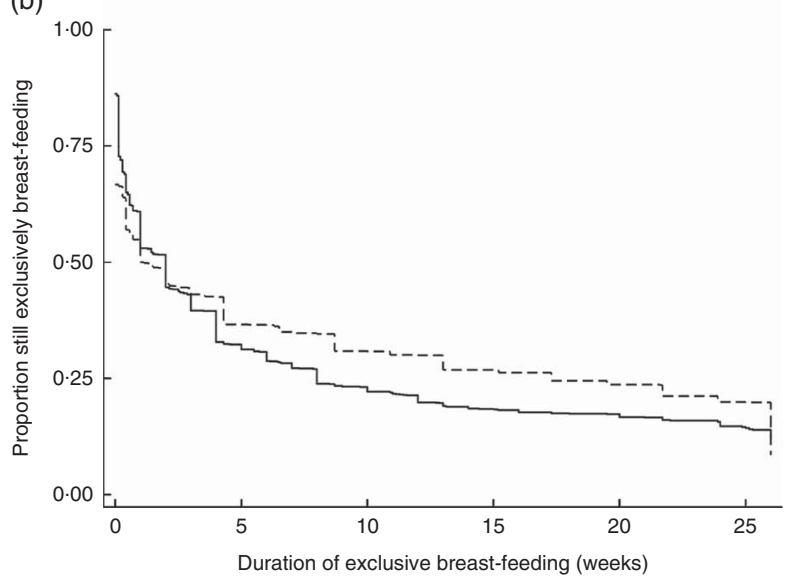

Fig. 3 Kaplan-Meier survival estimates of the duration of (a) any breast-feeding and (b) exclusive breast-feeding by study cohort (- cohort $1, n$ 1320; - - - - - cohort 2, n 1240). The two cohorts of breast-feeding mother-infant pairs were recruited from in-patient postnatal units of four public hospitals in Hong Kong, before (cohort 1) and after (cohort 2) implementation of the policy to stop accepting free infant formula; $P<0.001$ for (a), $P=0.58$ for (b) of obtaining BFHI accreditation. Thus, it is possible that the increased awareness about the reasons for the policy and the negative consequences of indiscriminate

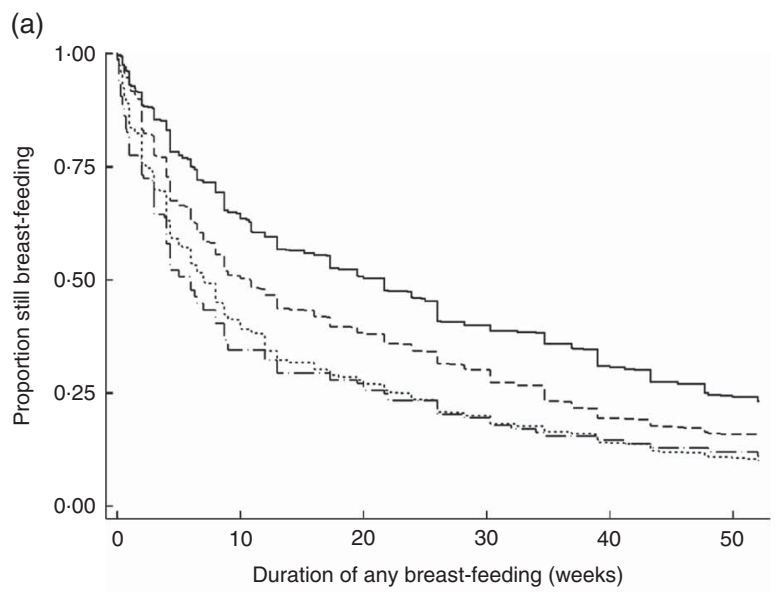

(b)

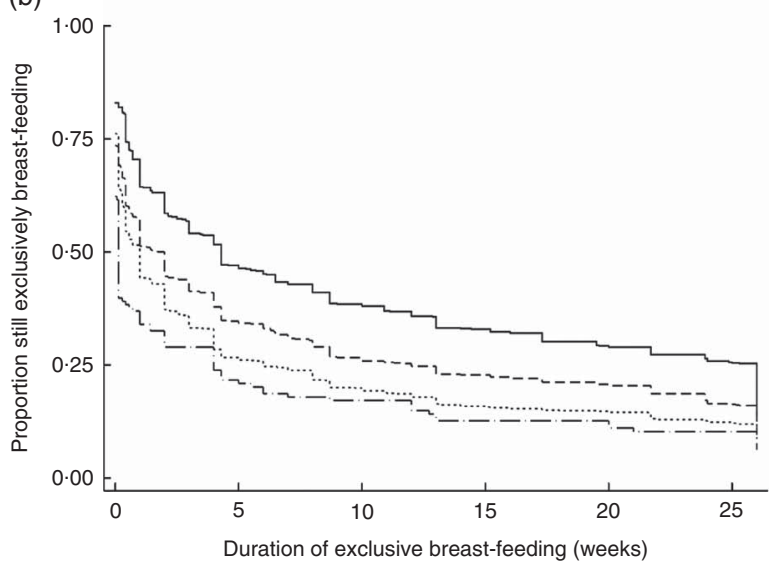

Fig. 4 Kaplan-Meier survival estimates of the duration of (a) any breast-feeding and (b) exclusive breast-feeding by the amount of in-hospital breast-feeding ( - , exclusive; ----- , highpartial; . . . . . medium-partial; _ . _ . - low-partial). Pooled sample of two cohorts of breast-feeding mother-infant pairs, who were recruited from in-patient postnatal units of four public hospitals in Hong Kong, before and after implementation of the policy to stop accepting free infant formula; both $P<0.001$ (trend)

Table 2 Association between study cohort and duration of any and exclusive breast-feeding among two study cohorts of breast-feeding mother-infant pairs ( $n$ 2560), who were recruited from in-patient postnatal units of four public hospitals in Hong Kong, before (cohort 1 ) and after (cohort 2) implementation of the policy to stop accepting free infant formula

\begin{tabular}{|c|c|c|c|c|c|c|c|c|}
\hline \multirow[b]{2}{*}{ Study cohort } & \multicolumn{2}{|c|}{ Unadjusted } & \multicolumn{2}{|c|}{ Model $1^{*}$} & \multicolumn{2}{|c|}{ Model $2 \dagger$} & \multicolumn{2}{|c|}{ Model 3‡ } \\
\hline & $\mathrm{HR}$ & $95 \% \mathrm{Cl}$ & $\mathrm{HR}$ & $95 \% \mathrm{Cl}$ & $\mathrm{HR}$ & $95 \% \mathrm{Cl}$ & $\mathrm{HR}$ & $95 \% \mathrm{Cl}$ \\
\hline \multicolumn{9}{|c|}{ Any breast-feeding } \\
\hline Cohort 1 & 1.00 & Ref. & 1.00 & Ref. & 1.00 & Ref. & 1.00 & Ref. \\
\hline Cohort 2 & 0.81 & $0.74,0.88$ & 0.82 & $0.74,0.90$ & 0.79 & $0.72,0.87$ & 0.81 & $0.73,0.90$ \\
\hline \multicolumn{9}{|c|}{ Exclusive breast-feeding } \\
\hline Cohort 1 & 1.00 & Ref. & 1.00 & Ref. & 1.00 & Ref. & 1.00 & Ref. \\
\hline Cohort 2 & 0.98 & $0.91,1.06$ & 0.99 & $0.91,1.08$ & 0.98 & $0.90,1.07$ & 1.01 & $0.93,1.11$ \\
\hline
\end{tabular}

HR, hazard ratio; Ref., referent category.

*Adjusted for maternal age, maternal education, family income, length of residence in Hong Kong, returning to work and study site.

†Further adjusted for parity, childbirth class attendance, breast-feeding class attendance and delivery type.

fFurther adjusted for intention to exclusively breast-feed, participant's own breast-feeding status, previous breast-feeding experience and husband's infant feeding preference. 
Table 3 Association between in-hospital proportion of breast-feeding and duration of any and exclusive breast-feeding; pooled sample of two cohorts of breast-feeding mother-infant pairs ( $n$ 2560), who were recruited from in-patient postnatal units of four public hospitals in Hong Kong, before (cohort 1) and after (cohort 2) implementation of the policy to stop accepting free infant formula

\begin{tabular}{|c|c|c|c|c|c|c|c|c|c|}
\hline \multirow[b]{2}{*}{ Amount of in-hospital breast-feeding } & \multirow[b]{2}{*}{ Prevalence (\%) } & \multicolumn{2}{|c|}{ Unadjusted } & \multicolumn{2}{|c|}{ Model $1^{\star}$} & \multicolumn{2}{|c|}{ Model 2† } & \multicolumn{2}{|c|}{ Model 3‡ } \\
\hline & & HR & $95 \% \mathrm{Cl}$ & $\mathrm{HR}$ & $95 \% \mathrm{Cl}$ & $\mathrm{HR}$ & $95 \% \mathrm{Cl}$ & $\mathrm{HR}$ & $95 \% \mathrm{Cl}$ \\
\hline \multicolumn{10}{|l|}{ Any breast-feeding } \\
\hline Exclusive & $29 \cdot 1$ & 1.00 & Ref. & 1.00 & Ref. & 1.00 & Ref. & 1.00 & Ref. \\
\hline High-partial & $27 \cdot 5$ & 1.34 & $1.20,1.51$ & 1.34 & $1 \cdot 19,1.51$ & 1.34 & $1.19,1.51$ & 1.29 & $1.14,1.46$ \\
\hline Medium-partial & 37.9 & 1.75 & $1.57,1.95$ & 1.75 & $1.56,1.96$ & 1.79 & $1.59,2.02$ & 1.68 & $1.49,1.90$ \\
\hline Low-partial & 5.5 & 1.86 & $1.53,2.27$ & 1.81 & $1 \cdot 48,2 \cdot 21$ & 1.97 & $1.58,2.44$ & 1.73 & $1 \cdot 39,2 \cdot 16$ \\
\hline \multicolumn{10}{|l|}{ Exclusive breast-feeding } \\
\hline Exclusive & 29.1 & 1.00 & Ref. & 1.00 & Ref. & 1.00 & Ref. & 1.00 & Ref. \\
\hline High-partial & 27.5 & 1.26 & $1.14,1.40$ & 1.28 & $1 \cdot 15,1.42$ & 1.28 & $1.15,1.42$ & 1.22 & $1 \cdot 10,1.36$ \\
\hline Medium-partial & 37.9 & 1.44 & $1.31,1.58$ & 1.52 & $1.37,1.68$ & 1.56 & $1.40,1.73$ & 1.47 & $1.32,1.64$ \\
\hline Low-partial & 5.5 & 1.74 & $1.45,2.09$ & 1.80 & $1.49,2 \cdot 17$ & 1.91 & $1.56,2.33$ & 1.69 & $1.38,2.07$ \\
\hline
\end{tabular}

HR, hazard ratio; Ref., referent category.

${ }^{*}$ Adjusted for maternal age, maternal education, family income, length of residence in Hong Kong, returning to work, study site and study cohort. †Further adjusted for parity, childbirth class attendance, breast-feeding class attendance and delivery type.

fFurther adjusted for intention to exclusively breast-feed, participant's own breast-feeding status, previous breast-feeding experience and husband's infant feeding preference.

infant formula supplementation contributed more to the changes observed than the perceived financial incentives. However, removing free infant formula from hospitals communicates to all staff that the hospital supports breastfeeding. It also reinforces the message that infant formula is not harmless and that breast-feeding babies should only be provided with supplements when medically necessary. It is also important to note that removing the free supply of infant formula necessitates improving other hospital practices that support breast-feeding. In another separate study ${ }^{(56)}$, there was also substantial improvement in the early initiation of breast-feeding after the infant formula policy change. Although cause and effect cannot be established in observational studies such as this, we believe the findings of the current study do support the WHO position that the provision of free infant formula to hospitals encourages and promotes formula supplementation $^{(18)}$.

To the knowledge of the authors, the present study is the first one to specifically evaluate the effect of stopping free infant formula on in-hospital formula supplementation rates and breast-feeding duration. Participant dropout rates in the present study were low in both cohorts and breast-feeding follow-up data were collected on $95.4 \%$ of the sample. However, the present study has some limitations. First, participation was voluntary and it is possible that women who had a more positive view of breastfeeding were more willing to take part. Data on those who refused to participate, or the proportion of eligible mothers who chose not to participate, are not available. However, the pattern of refusal was observed to be similar in both cohorts. Second, breast-feeding continuation data were collected by maternal self-report and it is possible that mothers did not accurately report the duration of any or exclusive breast-feeding. The regular follow-up in the present study is likely to have minimized any recall bias and previous research has found that maternal reporting of breast-feeding initiation and duration is accurate for up to 20 years after the period of breast-feeding ${ }^{(57,58)}$. Third, the lack of a comparison group does limit the attribution of observed changes directly to the new formula policy. However, as the policy was implemented in all public and most private hospitals at the same time, recruiting participants for a comparison group was not possible. Finally, the study evaluated breast-feeding rates in two different time periods with a 5-year period between recruitment of the two study cohorts, which also limits the ability to attribute the changes in breast-feeding practices directly to the change in infant formula policy. Although implementation of the policy was first scheduled in 2007 , numerous delays meant that there was an unexpected extended interval between pre- and post-policy data collection. It is possible that breast-feeding practices may have improved naturally over this time period, independent of the policy change. However, breast-feeding data collected for another study ${ }^{(59)}$ from the same hospitals with the same participant selection criteria, immediately prior to the policy implementation, show that there was no change in breast-feeding duration in the time interval between planned and actual policy implementation (see online supplementary material, Supplemental Fig. 1). Furthermore, as previously highlighted, the delay in policy implementation did provide public hospital management and staff with more time to have in-depth discussions about the rationale for the policy, how to improve hospital breastfeeding practices and how to become more baby-friendly.

\section{Conclusion}

The present study shows that rates of formula supplementation were significantly reduced and breast-feeding 
duration increased after hospitals stopped accepting free infant formula from manufacturers. However, the duration of breast-feeding in Hong Kong mothers still remains far short of the recommended 6 months of exclusive breastfeeding and continued breast-feeding thereafter for up to 2 years. To further improve hospital practices, institutional commitment is required to ensure that all new mothers receive adequate postnatal breast-feeding support consistent with WHO guidelines. Evidence-based guidelines on indicators for introducing infant formula that are consistent with the BFHI ten steps will also help to further decrease the rates of formula supplementation to expected levels.

\section{Acknowledgements}

Financial support: This study was supported by the Health and Medical Research Fund (grant number 05060721) from the Food and Health Bureau, Government of the Hong Kong Special Administration Region; the University of Hong Kong (grant \#10207306); the University of Hong Kong's Strategic Research Theme of Public Health; and the School of Nursing of the University of Hong Kong. The funders had no role in the design, analysis or writing of this article. Conflict of interest: None. Authorship: M.T. conceptualized the study, obtained funding, oversaw the implementation of the study, conducted data analysis, co-wrote the first draft of the manuscript, critically reviewed and revised the manuscript, and approved the final manuscript as submitted. K.Y.W.L. assisted with data analysis, co-wrote the first draft of the manuscript, critically reviewed and revised the manuscript, and approved the final manuscript as submitted. D.Y.T.F. contributed to the study design, assisted with data analysis, critically reviewed and revised the manuscript, and approved the final manuscript as submitted. I.L.Y.L. contributed to the study design, facilitated participant recruitment, critically reviewed and revised the manuscript, and approved the final manuscript as submitted. A.S. contributed to the study design, facilitated participant recruitment, critically reviewed and revised the manuscript, and approved the final manuscript as submitted. C.L. contributed to the study design, facilitated participant recruitment, critically reviewed and revised the manuscript, and approved the final manuscript as submitted. K.M.W. coordinated and supervised data collection, assisted with data analysis, critically reviewed and revised the manuscript, and approved the final manuscript as submitted. D.L.B. assisted with data analysis, critically reviewed and revised the manuscript, and approved the final manuscript as submitted. K.L.W. assisted with data collection and study management, critically reviewed and revised the manuscript, and approved the final manuscript as submitted. E.M.Y.W. contributed to the study design, critically reviewed and revised the manuscript, and approved the final manuscript as submitted. N.P.T.C. assisted with data analysis, critically reviewed and revised the manuscript, and approved the final manuscript as submitted. J.E.D. contributed to the study design, critically reviewed and revised the manuscript, and approved the final manuscript as submitted. Ethics of human subject participation: This study was conducted according to the guidelines laid down in the Declaration of Helsinki and all procedures involving human subjects/patients were approved by the Institutional Review Board of the University of Hong Kong/Hospital Authority Hong Kong West Cluster and by all of the participating institutions. All participants gave informed written consent.

\section{Supplementary material}

To view supplementary material for this article, please visit http://dx.doi.org/10.1017/S1368980015000117

\section{References}

1. American Academy of Pediatrics (2012) Policy statement: breastfeeding and the use of human milk. Pediatrics 129, e827-e841.

2. Ip S, Chung M, Raman G et al. (2007) Breastfeeding and maternal and infant health outcomes in developed countries. Evid Rep Technol Assess (Full Rep) issue 153, $1-186$.

3. Quigley MA, Kelly YJ \& Sacker A (2007) Breastfeeding and hospitalization for diarrheal and respiratory infection in the United Kingdom Millennium Cohort Study. Pediatrics 119, e837-e842.

4. Tarrant M, Kwok MK, Lam TH et al. (2010) Breast-feeding and childhood hospitalizations for infections. Epidemiology 21, 847-854.

5. Centers for Disease Control and Prevention (2013) Breastfeeding Report Card - United States, 2013. Atlanta, GA: CDC.

6. Organisation for Economic Co-operation and Development (2009) OECD Family database: breastfeeding rates. http:// www.oecd.org/social/family/43136964.pdf (accessed July 2013).

7. Biro MA, Sutherland GA, Yelland JS et al. (2011) In-hospital formula supplementation of breastfed babies: a populationbased survey. Birth 38, 302-310.

8. Chantry CJ, Dewey KG, Peerson JM et al. (2014) In-hospital formula use increases early breastfeeding cessation among first-time mothers intending to exclusively breastfeed. J Pediatr 164, 1339-1345.

9. Gagnon AJ, Leduc G, Waghorn K et al. (2005) In-hospital formula supplementation of healthy breastfeeding newborns. J Hum Lact 21, 397-405.

10. Holmes AV, Auinger P \& Howard CR (2011) Combination feeding of breast milk and formula: evidence for shorter breast-feeding duration from the National Health and Nutrition Examination Survey. J Pediatr 159, 186-191.

11. Parry JE, Ip DK, Chau PY et al. (2013) Predictors and consequences of in-hospital formula supplementation for healthy breastfeeding newborns. J Hum Lact 29, $527-536$

12. Tender JA, Janakiram J, Arce E et al. (2009) Reasons for in-hospital formula supplementation of breastfed infants from low-income families. J Hum Lact 25, 11-17. 
13. McDonald SD, Pullenayegum E, Chapman B et al. (2012) Prevalence and predictors of exclusive breastfeeding at hospital discharge. Obstet Gynecol 119, 1171-1179.

14. Academy of Breastfeeding Medicine Protocol Committee (2009) ABM clinical protocol \#3: hospital guidelines for the use of supplementary feedings in the healthy term breastfed neonate, revised 2009. Breastfeed Med 4, 175-182.

15. World Health Organization (1998) Evidence for the Ten Steps to Successful Breastfeeding. WHO/CHD/98.9. Geneva: WHO.

16. Kaplan DL \& Graff KM (2008) Marketing breastfeeding reversing corporate influence on infant feeding practices. J Urban Health 85, 486-504.

17. World Health Organization \& UNICEF (1989) Protecting, Promoting, and Supporting Breastfeeding: The Special Role of Maternity Services. Geneva: WHO and UNICEF.

18. World Health Organization \& UNICEF (2009) Baby-Friendly Hospital Initiative: Revised, Updated and Expanded for Integrated Care. Geneva: WHO and UNICEF.

19. DiGirolamo AM, Grummer-Strawn LM \& Fein SB (2008) Effect of maternity-care practices on breastfeeding. Pediatrics 122, Suppl. 2, S43-S49.

20. Tarrant M, Wu KM, Fong DY et al. (2011) Impact of babyfriendly hospital practices on breastfeeding in Hong Kong. Birth 38, 238-245.

21. Merten S, Dratva J \& Ackermann-Liebrich U (2005) Do baby-friendly hospitals influence breastfeeding duration on a national level? Pediatrics 116, e702-e708.

22. Rosenberg KD, Eastham CA, Kasehagen LJ et al. (2008) Marketing infant formula through hospitals: the impact of commercial hospital discharge packs on breastfeeding. $A m J$ Public Health 98, 290-295.

23. Feldman-Winter L, Grossman X, Palaniappan A et al. (2012) Removal of industry-sponsored formula sample packs from the hospital: does it make a difference? J Hum Lact $\mathbf{2 8}$, 380-388.

24. Perez-Escamilla R, Pollitt E, Lonnerdal B et al. (1994) Infant feeding policies in maternity wards and their effect on breast-feeding success: an analytical overview. Am J Public Health 84, 89-97.

25. Baby Friendly Hospital Initiative (1992) UNICEF Guidelines: Part IV. Ending the Distribution of Free and Low-Cost Supplies of Infant Formula to Health Care Facilities: Assisting Government Action and Gaining Industry Commitment. Geneva: UNICEF.

26. Haiek LN (2012) Compliance with baby-friendly policies and practices in hospitals and community health centers in Quebec. J Hum Lact 28, 343-358.

27. Venancio SI, Saldiva SR, Escuder MM et al. (2012) The Baby-Friendly Hospital Initiative shows positive effects on breastfeeding indicators in Brazil. J Epidemiol Community Health 66, 914-918.

28. Hawkins SS, Stern AD, Baum CF et al. (2014) Compliance with the Baby-Friendly Hospital Initiative and impact on breastfeeding rates. Arch Dis Child Fetal Neonatal Ed 99, F138-F143.

29. Rosenberg KD, Stull JD, Adler MR et al. (2008) Impact of hospital policies on breastfeeding outcomes. Breastfeed Med 3, 110-116.

30. Hawkins SS, Stern AD, Baum CF et al. (2015) Evaluating the impact of the Baby-Friendly Hospital Initiative on breastfeeding rates: a multi-state analysis. Public Health Nutr 18, 189-197.

31. Baby Friendly Hospital Initiative Hong Kong Association (2013) World Breastfeeding Week 2013: Annual Summary. Hong Kong: Baby Friendly Hospital Initiative Hong Kong Association.

32. Tarrant M, Fong DY, Wu KM et al. (2010) Breastfeeding and weaning practices among Hong Kong mothers: a prospective study. BMC Pregnancy Childbirth 10, 27.
33. Kwan D \& Leung E (2011) Vital statistics in 2010. Public Health Epidemiol Bull 20, 15-30.

34. Panel on Health Services (2012) Latest Arrangement for Non-local Pregnant Women Giving Birth in Hong Kong. LC Paper no. CB(2)1863/11-12(01). Hong Kong: Panel on Health Services.

35. LaFraniere S (2012) Mainland Chinese flock to Hong Kong to give birth. The New York Times, 22 February; available at http://www.nytimes.com/2012/02/23/world/asia/mainlandchinese-flock-to-hong-kong-to-have-babies.html?pagewanted= all\&_r=0

36. World Health Organization (1991) Indicators for Assessing Breast-Feeding Practices. Geneva: WHO.

37. Labbok M \& Krasovec K (1990) Toward consistency in breastfeeding definitions. Stud Fam Plann 21, 226-230.

38. Perrine CG, Scanlon KS, Li R et al. (2012) Baby-Friendly hospital practices and meeting exclusive breastfeeding intention. Pediatrics 130, 54-60.

39. Dodgson JE, Tarrant M, Fong DYT et al. (2003) Breastfeeding patterns of primiparous mothers in Hong Kong. Birth 30, 195-202.

40. Kramer MS, Chalmers B, Hodnett ED et al. (2001) Promotion of Breastfeeding Intervention Trial (PROBIT): a randomized trial in the Republic of Belarus. JAMA 285, 413-420.

41. Cleves M, Gould W, Gutierrez RG et al. (2010) An Introduction to Survival Analysis Using Stata, 3rd ed. College Station, TX: Stata Press.

42. Cox DR \& Oakes D (1984) Analysis of Survival Data. London: Chapman and Hall.

43. Rothman KJ, Greenland S \& Lash TL (2012) Modern Epidemiology, 3rd ed. Philadelphia, PA: Lippincott, Williams and Wilkins

44. DiGirolamo A, Thompson N, Martorell $\mathrm{R}$ et al. (2005) Intention or experience? Predictors of continued breastfeeding. Health Educ Behav 32, 208-226.

45. Donath SM, Amir LH \& Team AS (2003) Relationship between prenatal infant feeding intention and initiation and duration of breastfeeding: a cohort study. Acta Paediatr $\mathbf{9 2}$, 352-356.

46. Declercq E, Labbok MH, Sakala C et al. (2009) Hospital practices and women's likelihood of fulfilling their intention to exclusively breastfeed. Am J Public Health 99, 929-935.

47. Fu I, Fong D, Heys M et al. (2014) Professional breastfeeding support for first-time mothers: a multicentre cluster randomised controlled trial. BJOG 121, 1673-1683.

48. Tarrant M, Dodgson JE \& Wu KM (2014) Factors contributing to early breastfeeding cessation among Chinese mothers: an exploratory study. Midwifery 30, 1088-1095.

49. Tarrant M, Dodgson JE \& Tsang SF (2002) Initiating and sustaining breastfeeding in Hong Kong: contextual influences on new mothers' experiences. Nurs Health Sci $\mathbf{4}$, 189-191.

50. Wright A, Rice S \& Wells S (1996) Changing hospital practices to increase the duration of breastfeeding. Pediatrics 97, 669-675.

51. Fairbank L, O'Meara S, Renfrew MJ et al. (2000) A systematic review to evaluate the effectiveness of interventions to promote the initiation of breastfeeding. Health Technol Assess 4, 1-171.

52. Howard CR, Howard FM, Lanphear B et al. (2003) Randomized clinical trial of pacifier use and bottle-feeding or cupfeeding and their effect on breastfeeding. Pediatrics 111, 511-518.

53. Gray-Donald K, Kramer MS, Munday S et al. (1985) Effect of formula supplementation in the hospital on the duration of breast-feeding: a controlled clinical trial. Pediatrics $\mathbf{7 5}$, 514-518.

54. World Health Organization \& UNICEF (2009) Acceptable Medical Reasons for Use of Breast-milk Substitutes. WHO/ NMH/NHD/09.01; WHO/FCH/CAH/09.01. Geneva: WHO. 
55. Berry NJ \& Gribble KD (2008) Breast is no longer best: promoting normal infant feeding. Matern Child Nutr 4, 74-79.

56. Tarrant M, Fong DYT, Lee IL et al. (2014) Changing Infant Feeding Models: Impact of Cessation of Complimentary Infant Formula in Public Hospitals on the Duration and Exclusivity of Breastfeeding. Final Report Submitted to the Grant Review Board of the Health and Medical Research Fund, Government of the Hong Kong SAR. Hong Kong: Food and Health Bureau, Government of the Hong Kong SAR.
57. Li R, Scanlon KS \& Serdula MK (2005) The validity and reliability of maternal recall of breastfeeding practice. Nutr Rev 63, 103-110.

58. Natland ST, Andersen LF, Nilsen TI et al. (2012) Maternal recall of breastfeeding duration twenty years after delivery. BMC Med Res Methodol 12, 179.

59. Tarrant M, Wu KM, Yuen CY et al. (2013) Determinants of $2009 \mathrm{~A} / \mathrm{H} 1 \mathrm{~N} 1$ influenza vaccination among pregnant women in Hong Kong. Matern Child Health J 17, 23-32. 